\title{
Disturbance Rejection in Multi-Rotor Unmanned Aerial Vehicles Using a Novel Rotor Geometry
}

\author{
Florentin von Frankenberg, Scott Nokleby \\ Mechatronic and Robotic Systems Laboratory \\ University of Ontario Institute of Technology \\ Oshawa, Ontario, Canada \\ florentin.vonfrankenberg@uoit.ca; scott.nokleby@uoit.ca
}

\begin{abstract}
This paper presents a novel multi-rotor Unmanned Aerial Vehicle (UAV). The UAV, or OmniCopter, has eight rotors: four lift rotors in the typical quadcopter layout and four thrust motors that are orthogonal to the lift rotors and arranged as two pairs of coaxial counter-rotating rotors. The OmniCopter layout allows the decoupling of translation and orientation that is found in traditional multi-rotor UAVs. The OmniCpoter is able to translate in the plane orthogonal to the lift rotors without having to first roll and/or pitch. Testing is undertaken to determine the OmniCopters ability to reject disturbances from gusting winds. The results show that the OmniCopter has better disturbance rejection abilities when compared to the traditional multi-rotor layout.
\end{abstract}

Keywords: Unmanned Aerial Vehicle (UAV); Disturbance Rejection; Wind Gusts; OmniCopter

\section{Introduction}

Unmanned Aerial Vehicles (UAVs) are now common place for inspection tasks. However, there has been limited work on utilizing UAVs for tasks such as construction and maintenance. For such tasks, UAVs would be required to interact with the environment via a robotic manipulator or other similar device.

As noted in [1], in order for UAVs to interact with their environment there must be force closure. In other words, the base of the robot manipulator, namely the UAV, must be able to resist the wrench generated by the motion of the manipulator. Unlike terrestrial-based mobile manipulators, aerial manipulators cannot rely on friction to allow them to resist these wrenches. This poses a number of challenges for aerial manipulation.

In traditional multi-rotor vehicles, all the rotors are parallel to one another. In such an arrangement, it is impossible to generate linear forces in the plane perpendicular to the axes of the rotors. Traditional multi-rotors can only pitch, roll, yaw, and lift. In order to move in the horizontal plane, a traditional multi-rotor must first pitch and/or roll. This means that these systems are nonholonomic. The same holds true for helicopters. This inability to generate forces in the plane perpendicular to the axes of the rotors make traditional multi-rotors and helicopters unsuitable for mobile manipulation as noted in [2].

There have been attempts in the research community to address some of these limitations. In [3], a single actuator was added to a traditional quadcopter design to allow their UAV to apply a horizontal force to vertical surfaces while in flight. A novel hexrotor design was developed in [1]. In order to generate off-axis forces, the rotors' axes of thrust are canted by $30^{\circ}$ such that they are no longer parallel. This is very similar to [4] except that in [4] the rotors are canted at $45^{\circ}$ and variable pitch rotors are used as opposed to variable speed ones. In [5], a UAV with five rotors is presented. The design features two central counter-rotating coaxial rotors responsible for generating the majority of the lift with three outer rotors used for maneuvering.

In [6], an eight rotor UAV was developed with four rotors added outwards of a standard quadcopter design. The additional four rotors face perpendicular to the original four quadcopter rotors. The resulting system has translational and rotational dynamics almost completely decoupled, but there is some aerodynamic interaction between the rotors due to the fact that the horizontally facing rotors direct an airstream directly underneath the lifting rotors. In [7], a fully omnidirectional UAV with eight rotors is presented. The geometric configuration of rotors was chosen to maximize agility in any direction. Testing showed the system's ability to rotate about an axis independently of translational movement.

In [8], the authors of this current work presented a novel omni-directional quadcopter, dubbed the OmniCopter, that features a set of four thrust rotors orthogonal to the four main lift rotors. The concept is similar to that of [6] except the 
layout is more compact since the additional rotors are not directly outside of the lift rotors, but are located inside between the lift rotors. This layout eliminates the problems the design in [6] had with the interaction between the airflow of the thrust rotors with the lift rotors. In [8], preliminary testing of the OmniCopter is presented. In [9] the results of inclined landing tests of the OmniCopter are shown. In this work, the ability of the OmniCopter to resist disturbances, such as gusting winds is tested.

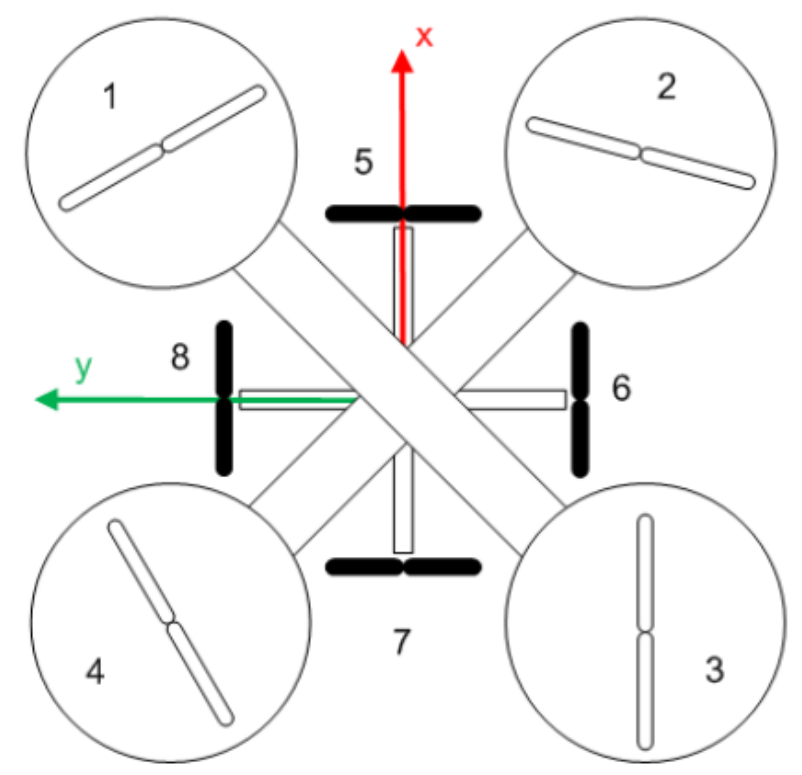

Fig. 1: OmniCopter rotor layout.

The outline of the remainder of the paper is as follows. Section 2 presents a brief overview of the OmniCopter. Section 3 discusses the control system used in this work. Section 4 presents the test results and a discussion of the results. The paper finishes with conclusions and recommendations for future work in Section 5.

\section{The OmniCopter}

The OmniCopter consists of a traditional quadcopter layout with the addition of four orthogonal thrust rotors. Figure 1 shows the rotor layout of the OmniCopter. The axes of the thrust rotors pass through the centre of gravity of the OmniCopter. By ensuring that each pair of co-axial thrust rotors rotate at the same speed, but opposite directions, the torques generated by the thrust rotors cancel each other out.

The equations of motion of the OmniCopter were developed by extending the equations of motion of a quadcopter, developed in [10], to include the additional thrust rotors as shown in [9]. The equations developed in [9] are presented here for the purposes of completeness.

Using the body-frame co-ordinate system and rotor numbering as shown in Figure 1, the torque in the body frame $(\tau \mathrm{B})$ is determined as:

$$
\tau_{B}=\mathbf{R}_{Z}\left(45^{\circ}\right)\left[\begin{array}{c}
L k\left(\omega_{1}^{2}-\omega_{3}^{2}\right) \\
L k\left(\omega_{2}^{2}-\omega_{4}^{2}\right) \\
b\left(\omega_{1}^{2}-\omega_{2}^{2}+\omega_{3}^{2}-\omega_{4}^{2}\right)
\end{array}\right]+\left[\begin{array}{c}
k_{t}\left(\omega_{7}\left|\omega_{7}\right|-\omega_{5}\left|\omega_{5}\right|\right) \\
k_{t}\left(\omega_{8}\left|\omega_{8}\right|-\omega_{6}\left|\omega_{6}\right|\right) \\
0
\end{array}\right]
$$

where $\mathbf{R}_{\mathrm{Z}}$ rotates the original body-torque matrix used from [10] about the $\mathrm{Z}$ axis to align it with the coordinate system shown in Figure $1, \omega_{i}$ is the angular velocity of the $i^{\text {th }}$ rotor, $k$ and $k_{t}$ are constants of proportionality accounting for motor and propeller properties for the main rotors and orthogonal thrust rotors, respectively, $L$ is the distance from the centre of mass to the centre of one of the main lift rotors, and $b$ is a proportionality constant which accounts for the properties of the motors and propellers as they relate to torque. 
The thrust force in the body-frame $\left(\mathbf{F}_{B}\right)$ is given as:

$$
\mathbf{F}_{B}=\left[\begin{array}{c}
k_{f}\left(\omega_{5}\left|\omega_{5}\right|+\omega_{7}\left|\omega_{7}\right|\right) \\
k_{f}\left(\omega_{6}\left|\omega_{6}\right|+\omega_{8}\left|\omega_{8}\right|\right) \\
k \sum_{i=1}^{4} \omega_{i}^{2}
\end{array}\right]
$$

where $k_{f}$ is a coefficient that relates the propellor speed to thrust. The drag force $\left(\mathbf{F}_{D}\right)$ is proportional to the vehicle's air velocity and is given as:

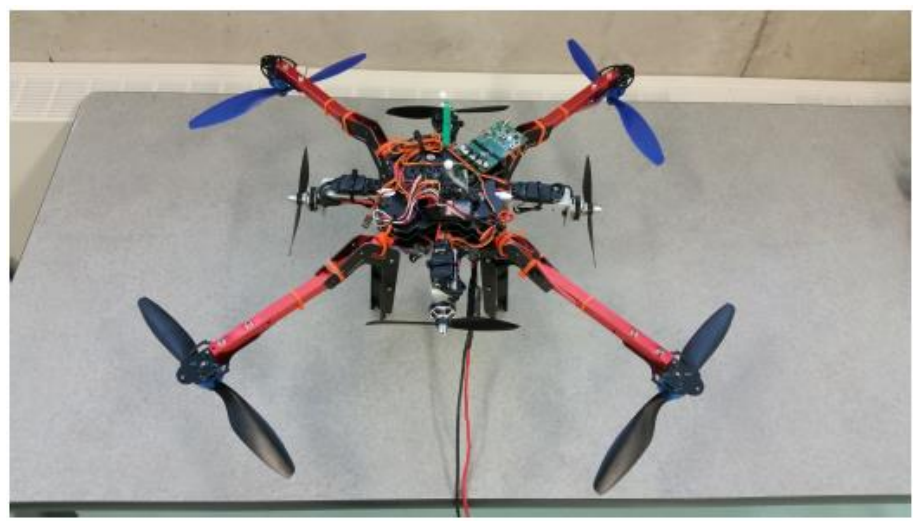

Fig. 2: The OmniCopter prototype.

$$
\mathbf{F}_{D}=\left[\begin{array}{l}
-k_{d} \dot{x} \\
-k_{d} \dot{y} \\
-k_{d} \dot{z}
\end{array}\right]
$$

where $k_{d}$ is a drag coefficient and $\dot{x}, \dot{y}$, and $\dot{z}$ are the translational velocities of the quadcopter in the $X, Y$, and $Z$ directions, respectively. The equations of motion are therefore:

$$
m \ddot{\mathbf{x}}=\left[\begin{array}{c}
0 \\
0 \\
-m \mathbf{g}
\end{array}\right]+\mathbf{R F}_{B}+\mathbf{F}_{D}
$$

where $m$ is the mass of the quadcopter, $\ddot{\mathbf{x}}$ is the acceleration of the quadcopter, $\mathbf{g}$ is the acceleration due to gravity, and $\mathbf{R}$ is a rotation matrix relating the body frame to the inertial frame.

Figure 2 shows the OmniCopter prototype. As mentioned above, it was necessary for the each pair of co-axial thrust rotors to be rotating in opposite directions at the same speed to cancel the torques generated by the thrust rotors. To achieve this, the OmniCopter uses variable speed rotors and can reverse the direction of rotor rotation to reverse the direction of thrust. Each co-axial pair of thrust rotors is counter-rotating, and contains one clock-wise oriented propeller and one counterclockwise oriented propeller. Since the propellers have opposite orientations, thrust is generated in the same direction while the rotors spin in opposite directions.

If necessary, a torque could be generated if the rotors spin in the same direction, while a net-zero thrust is generated. The sum of rotation rates determines net torque while the difference in rotation rates determines net thrust. Combinations of rotor speeds can be selected in order to generate desired combinations of torque and thrust. For the purposes of the implementation here, the co-axial thrust rotors were always commanded to rotate in opposite directions at equal speed.

For flight tests, a $2 \mathrm{~m}$ long dual-conductor 14 AWG stranded copper cable was used to supply power from a battery located on the ground to the prototype. For futher details on the OmniCopter, the reader is referred to [9]. 


\section{Control System}

An overview of the Control Implementation is shown in Figure 3. A modified motion capture system, called the BlackTrax Tracking System, was used to measure the position of several infrared (IR) LEDs on the OmniCopter. The software element of the BlackTrax system runs on a PC called the Tracking PC. The measured LED positions are fed back to another PC, called the Control PC, over wired Ethernet LAN. The Control PC calculates the control signals, which are then sent via USB to the Serial PPM (Pulse Position Modulation) Converter. The Serial PPM Converter converts the control signals from USB to PPM. The control signals are then fed into the RC (Radio Control) Transmitter Module. The RC Transmitter Module transmits the control signals over a $2.4 \mathrm{GHz}$ wireless radio protocol. The RC Transmitter Module also accepts manual control inputs from a human overseer. The human overseer can allow the control signals from the Control PC to pass through the RC Transmitter Module by activating a foot switch, or take manual control of the OmniCopter through the RC Transmitter Module's control joysticks. When the foot switch is released the UAV is under manual control. The OmniCopter receives the wireless control signals from the RC Transmitter Module and controls the velocity of its rotors. The OmniCopter rotor speeds influence its position and orientation. The Control PC is informed whether manual control is activated by the RC Transmitter

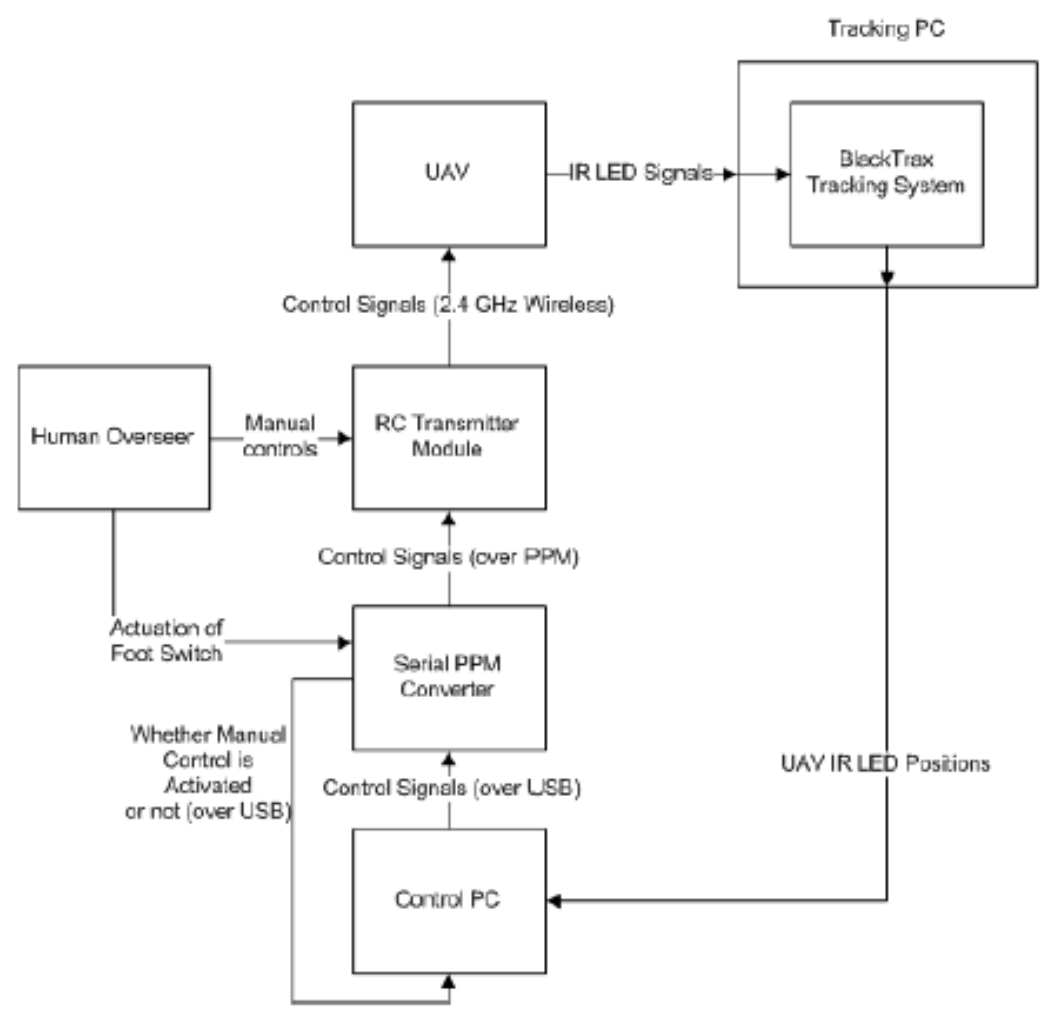

Fig. 3: Overview of the control implementation.

Module. This takes place over the same USB link as the control signals transfer over. It is shown using a separate arrow in the diagram for clarity.

The control system for the OmniCopter consists of nested PID (Proportional-Integral-Derivative) controllers. Control of orientation is independent of control of position, and uses two control loops, as depicted in Figure 4. The controller is implemented on a MultiWii Pro on-board flight controller that is available off-the-shelf and uses an Atmega $2560 \mathrm{MCU}$ (Micro-Controller Unit) as well as an on-board IMU (Inertial Measurement Unit) that consists of three orthogonal accelerometers and three orthogonal rate gyroscopes. The inner control loop handles roll, pitch, and yaw angular velocity and the outer control loop handles roll, pitch, and yaw angular position. The MultiWii Pro flight controller was not modified aside from tuning the PID parameters to suit the size and characteristics of the OmniCopter. 
Figure 5 depicts the control of position for the $X$ and $Y$ positions and Figure 6 depicts the control of the $Z$ position. The only difference between the two being that to control $Z$ position only the average thrust of the four main lift rotors is used, with no compensation for any roll or tilt angle. For both the control of the $X$ and $Y$ position and the control of the $Z$ position a nested control strategy is used in which the inner loop attempts to reach a velocity set-point, and the outer loop determines that velocity set-point. The velocity set-point is proportional to the position error. Additionally, after a velocity set-point is determined, it gets clamped to a reasonable maximum value so that a very large position error does not result in a very large velocity set-point. These maximum velocities were chosen based on the size of the test area and speeds within the OmniCopter's ability to accelerate to and decelerate safely within the test area. In practice, the clamping values used during experimentation and testing ranged from $10 \mathrm{~cm} / \mathrm{s}$ to $40 \mathrm{~cm} / \mathrm{s}$ for the $Z$ direction and $20 \mathrm{~cm} / \mathrm{s}$ to $100 \mathrm{~cm} / \mathrm{s}$ for the $X$ and $Y$ directions.

\section{Testing and Results}

To evaluate the OmniCopter's capacity for disturbance rejection it was compared against the traditional roll/pitch method of flight control. The intention was to evaluate whether or not the OmniCopter has advantages over a traditional multi-rotor. To do this, an autonomous controller using roll and pitch (without using the orthogonal thrusters at all) to control position was applied to the OmniCopter and this was compared against the orthogonal thruster control method. When using the orthogonal thrusters for control, a constant horizontal flight attitude was commanded.

First, a set of tests was conducted in calm conditions to set a performance benchmark. In these tests the OmniCopter was commanded to maintain a static hovering position and the deviations from the commanded position were recorded. The station-keeping test in calm conditions was done for both flight control methods. A second set of tests were conducted in simulated gusting winds induced by a large fan.

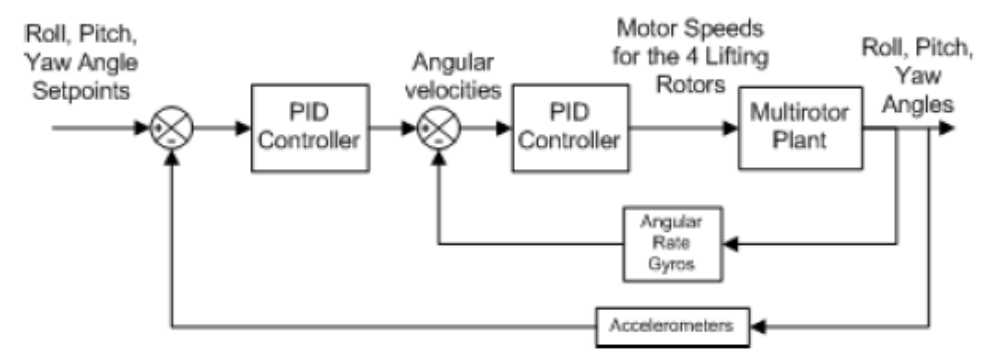

Fig. 4: Control for roll, pitch, and yaw.

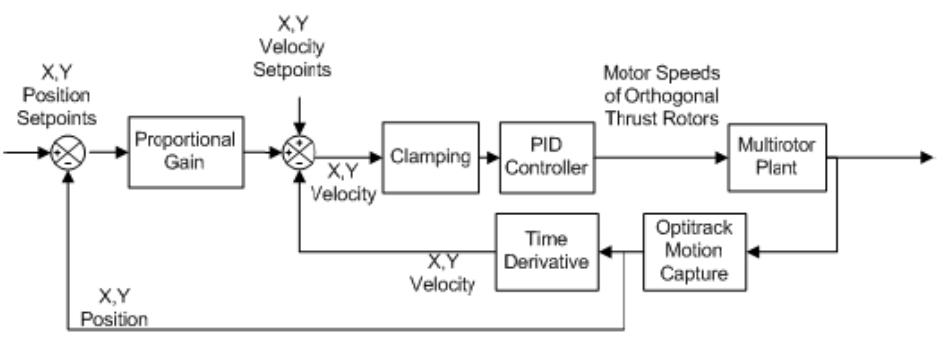

Fig. 5: Control for the $X$ and $Y$ positions.

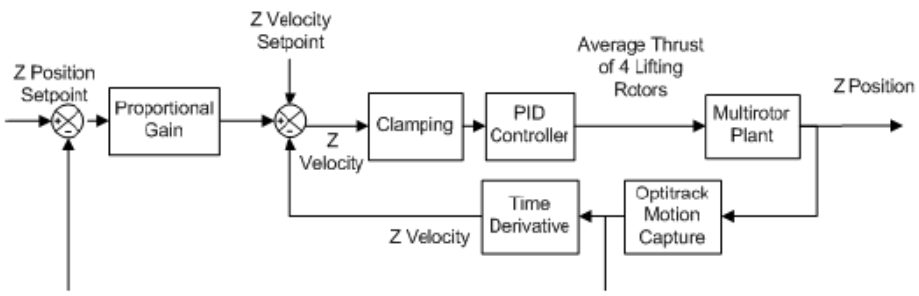

Fig. 6: Control for the $\mathrm{Z}$ position. 


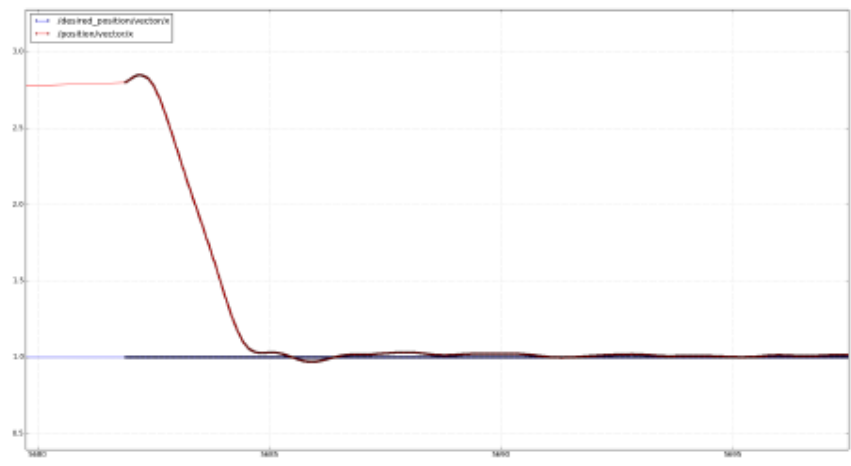

(a) Position vs. time.

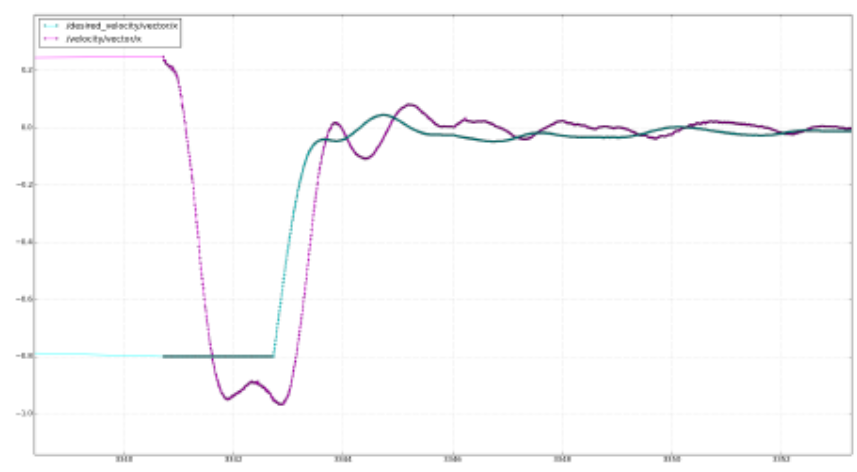

(b) Velocity vs. time

Fig. 7: Control using the traditional roll/pitch control method.

\subsection{Station-Keeping Benchmark Tests}

Figure 7 and Figure 8 show plots of the position and velocity in the $\mathrm{X}$ direction of the OmniCopter moving to and maintaining a static position under the roll/pitch control method and the orthogonal thruster control method, respectively. Figure 9 shows a side-by-side comparison of the two flight control methods. These are enlarged sections of the same tests once the OmniCopter reached the set-point. It can be seen in the plots that both flight control methods are able to maintain position within approximately $2 \mathrm{~cm}$ of the target position.

It is not entirely surprising that both flight control methods would perform equivalently in the absence of disturbances with the orthogonal thruster control method having smoother motion. The orthogonal thruster control method is expected to have an advantage in being capable of responding to disturbances more promptly, however, in the absence of disturbances other sources of error affect both flight control methods equally. Some potential sources of error are noise and inaccuracies in the position measurement system, latency in the data flow from sensing to actuation, and delay arising from the averaging filter used to estimate velocity from successive positions reported by the position measurement system.

\subsection{Station-Keeping in Gusting Winds}

In order to simulate gusting winds for these tests, a large fan was constructed using a powerful RC outrunner motor. An image of the fan mounted on a table can be seen in Figure 10. The fan was set to full power and an anemometer was used to measure air velocities at a point $2 \mathrm{~m}$ away from the fan. It was found that the fan generated a stream of air approximately $1 \mathrm{~m}$ wide with a fairly constant air velocity of $5 \mathrm{~m} / \mathrm{s}$ at the centre. Fluctuations of approximately $0.5 \mathrm{~m} / \mathrm{s}$ were observed at the centre of the air stream. As the anemometer was 


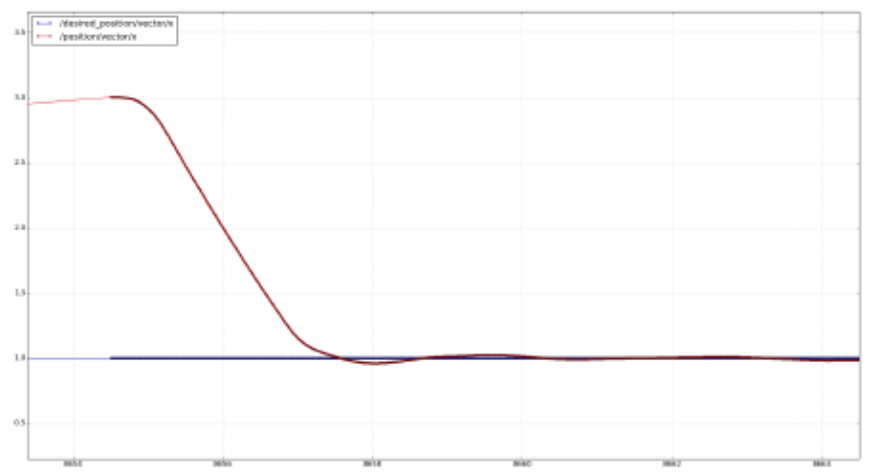

(a) Position versus time.

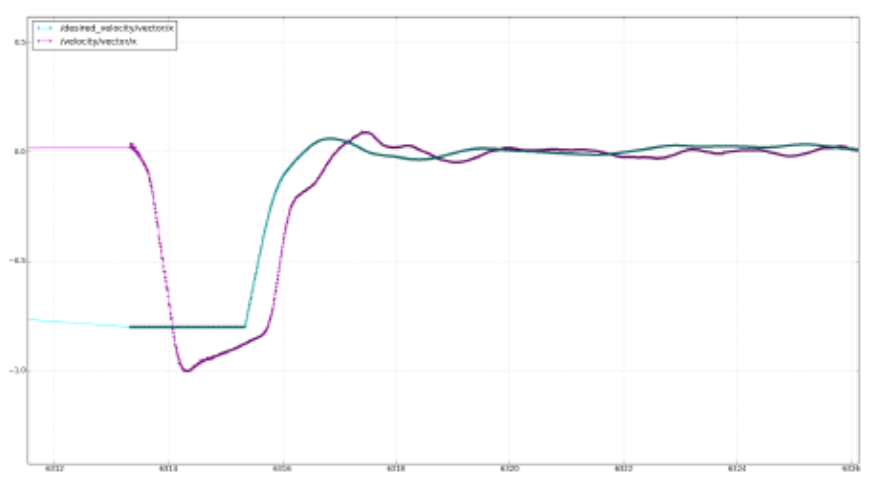

(b) Velocity versus time.

Fig. 8: Control using the orthogonal thruster control method.

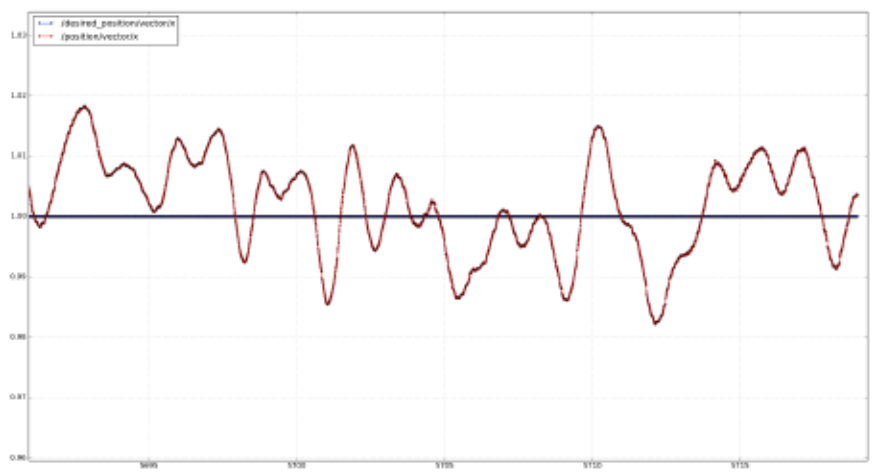

(a) Position versus time for traditional roll/pitch control method.

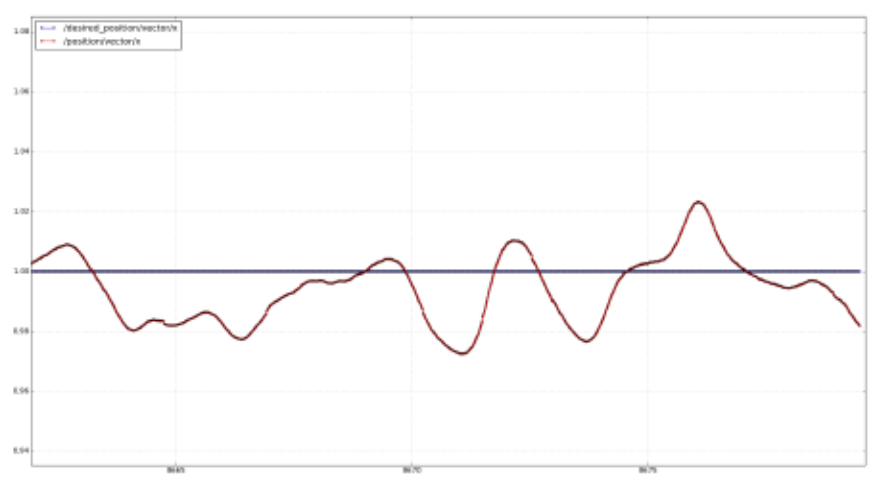

(b) Position versus time for orthogonal thruster control method.

Fig. 9: Enlarged sections of station keeping in calm conditions comparison test. 


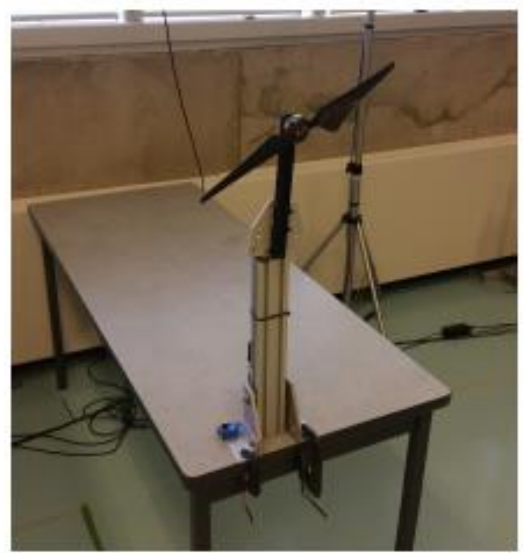

Fig. 10: The large fan used to simulate gusting winds.

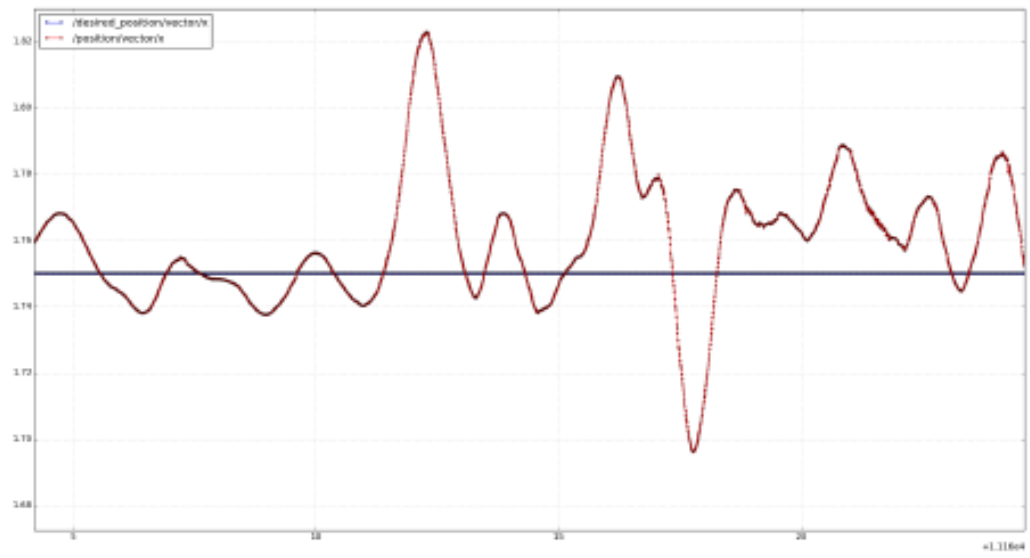

(a) Position versus time for traditional roll/pitch control method.

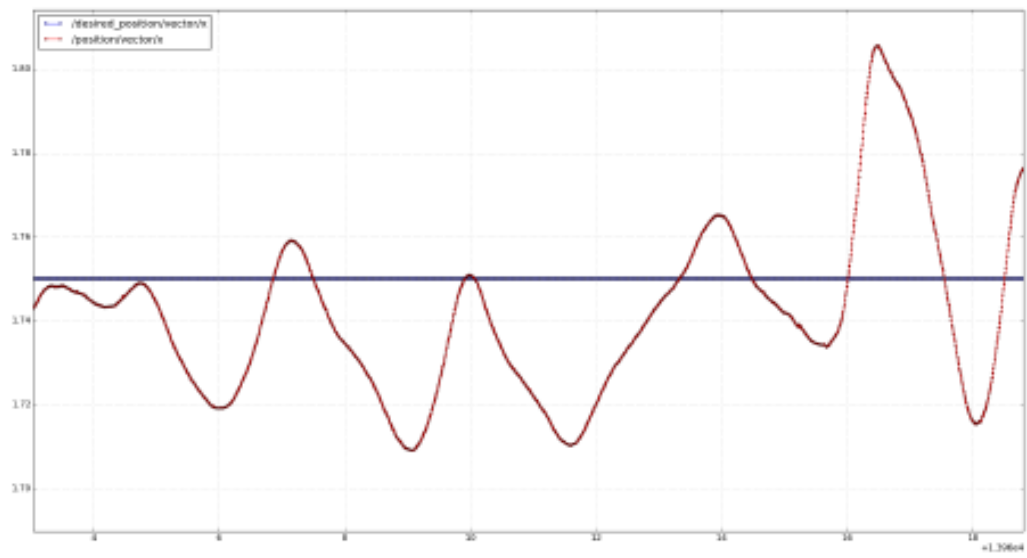

(b) Position versus time for orthogonal thruster control method.

Fig. 11: Station keeping in simulated gusting wind.

moved away from the centre, large fluctuations in air velocity were observed with velocities ranging from $2 \mathrm{~m} / \mathrm{s}$ to $5 \mathrm{~m} / \mathrm{s}$. At a point $50 \mathrm{~cm}$ from the centre of the air stream the air velocity was relatively constant at $1 \mathrm{~m} / \mathrm{s}$. This velocity gradient was useful for the intended station keeping test because if the OmniCopter gets pushed away from the goal position in the centre of the air stream, it experiences a sudden change in force. Generating rapidly changing aerodynamic forces and evaluating the OmniCopter performance was the goal for the test. 
For this test, the OmniCopter was commanded to fly to and maintain a position $2 \mathrm{~m}$ away from the fan in the centre of the air stream. Once the OmniCopter was maintaining the desired position the fan was manually engaged and the fan power alternated between full power and off. The fan was turned on and off once every two seconds to generate large time varying aerodynamic forces.

Figure 11 presents plots of position for the station keeping in the simulated wind test. The range in position seen in the plot for the traditional roll/pitch method is from slightly below $1.70 \mathrm{~cm}$ to slightly above $1.82 \mathrm{~cm}$. The range in position for the orthogonal thruster method is from $1.71 \mathrm{~cm}$ to $1.81 \mathrm{~cm}$. The maximum deflection from the setpoint for the roll/pitch method is $7 \mathrm{~cm}$ and $6 \mathrm{~cm}$ for the orthogonal thruster method.

Video of the tests, as well as the data, show that the thruster method resulted in smoother motion of the system when compared to the traditional method. The data indicates that the thruster method is better at disturbance rejection, although only slightly so.

It is expected that the choice of using variable speed rotors may be a limiting factor of the OmniCopter's performance. Tests were done on the thrust rates achievable by the thrust rotors using the Series 1580 Thrust Stand and Dynamometer from RCbenchmark.com. To measure the capability of the orthogonal thrusters to achieve a high rate of change of thrust, a square wave command input was given. This corresponded to alternating between large forward thrust and large reverse thrust. To achieve large changes in rotation speed, more motor torque is needed than would be for maintaining a constant rotation speed.

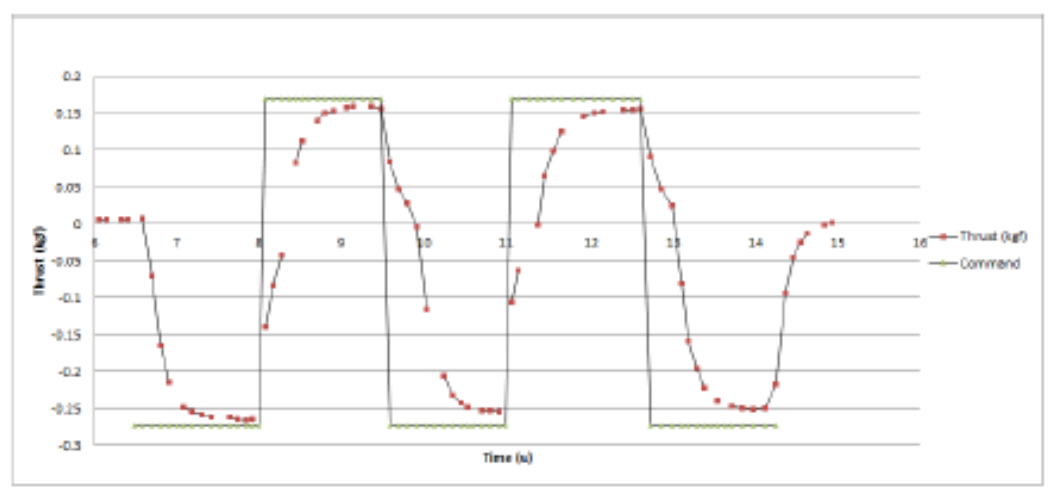

Fig. 12: Test for orthogonal thruster maximum rates of change.

The rotor's moment of inertia comes into play during changes in angular velocity. Figure 12 presents a plot of the orthogonal rotor thrust vs. time. For comparison purposes, a scaled and shifted plot of the command PWM (Pulse Width Modulation) input signal is overlaid over the values for thrust. It can be seen from the plot that it takes approximately 1 second for the rotor to switch from a large forward thrust to a large reverse thrust. It can also be seen that the magnitude of thrust is not symmetric for forward and reverse directions. This is because the propellers which were used are not designed for bidirectional rotation and are more efficient for one rotation direction. Because the orthogonal rotors are used in opposing pairs this asymmetry cancels out.

The thruster test results indicate that the motors are not capable of generating a compensatory thrust quickly enough to react to fast disturbances. Variable pitch rotors may offer greater improvements in performance as the ability to switch directions can be significantly faster. However, the use of variable pitch rotors will add additional mechanical complexity to the design of the system.

\section{Conclusions}

The ability of the OmniCopter to reject disturbances during flight was tested. The OmniCopter features a novel rotor layout: four lift rotors in the typical quadcopter layout and four thrust motors that are orthogonal to the lift rotors and arranged as two pairs of co-axial counter-rotating rotors. This layout allows the decoupling of translation and orientation, something that is impossible with traditional multi-rotor UAVs. The OmniCopter prototype was tested for its ability to resist unpredictable aerodynamic disturbances by generating simulated gusting winds with a large fan. It was found that in comparison to the traditional method of flight control using roll/pitch motions, the OmniCopter was able to resist disturbances better with the orthogonal thruster control method. 
In future, the use of variable pitch, larger diameter, and more powerful thrusters with stiffer propellers will be investigated to determine if further improvements in disturbance rejection can be achieved.

\section{Acknowledgements}

The authors would like to thank the Natural Sciences and Engineering Research Council (NSERC) of Canada for providing financial support of this work.

\section{References}

[1] G. Jiang, "Dextrous Hexrotor UAV Platform," M.S. thesis, University of Denver, 2013.

[2] R. Cano, C. Perez, F. Pruano, and A. Ollero, "Mechanical Design of a 6-DOF Aerial Manipulator for Assembling Bar Structures Using UAVs," in Proceedings of the 2nd IFAC Workshop on Research, Education, and Development of Unmanned Aerial Systems, 2013.

[3] A. Albers, S. Trautmann, T. Howard, T. A. Nguyen, M. Frietsch, and C. Sauter, "Semi-Autonomous Flying Robot for Physical Interaction with Environment," in Proceedings of the 2010 IEEE Conference on Robotics, Automation and Mechatronics, pp. 441-446, 2010.

[4] D. Langkamp, G. Roberts, A. Scillitoe, I. Lunnon, A. Llopis-Pascual, J. Zamecnik, S. Proctor, M. Rodriguez-Frias, M. Turner, A. Lanzon, and W. Crowther, "An Engineering Development of a Novel Hexrotor Vehicle for 3D Applications," in Proceedings of the International Micro Air Vehicles Conference, 2011.

[5] Y. Long and D. J. Cappelleri, "Omnicopter: A Novel Overactuated Micro Aerial Vehicle," in Advances in Mechanisms, Robotics and Design Education and Research, pp. 215-216, 2013.

[6] S. Salazar, H. Romero, R. Lozano, and P. Castillo, "Modeling and Real-Time Stabilization of an Aircraft Having Eight Rotors," Journal of Intelligent and Robotic Systems, vol. 54, no. 1-3, pp. 455-470, 2009.

[7] D. Brescianini and R. D'Andrea, "Design, Modeling and Control of Omni-Directional Aerial Robot," in Proceedings of the 2016 IEEE International Conference on Robotics and Automation, May 2016.

[8] F. von Frankenberg and S. B. Nokleby, "Development of the OmniCopter: A Highly Maneuverable Quad-Copter Platform for the Aerial Manipulator System," in Proceedings of the Fourth Joint International Conference on Multibody System Dynamics, Montreal, Canada, 2016.

[9] F. von Frankenberg and S. B. Nokleby, "Inclined Landing Testing of an Omni-Directional Unmanned Aerial Vehicle," in Proceedings of the 2017 CCToMM Symposium on Mechanisms, Machines, and Mechatronics, Montreal, Canada, May 2017.

[10] A. Gibiansky, “Quadcopter Dynamics, Simulation, and Control Introduction Quadcopter Dynamics,” pp. 1-18, 2012. [Online]. Available: http://andrew.gibiansky.com/blog/physics/quadcopter-dynamics/ 